\title{
Flow Components in a NaK Test Loop Designed to Simulate Conditions in a Nuclear Surface Power Reactor
}

\author{
Kurt A. Polzin* and Thomas J. Godfroy \\ NASA Marshall Space Flight Center \\ Propulsion Research and Technology Applications Branch / ER24, MSFC, AL 35812 \\ *256.544.5513, kurt.a.polzin@nasa.gov
}

\begin{abstract}
A test loop using $\mathrm{NaK}$ as the working fluid is presently in use to study material compatibility effects on various components that comprise a possible nuclear reactor design for use on the lunar surface. A DC electromagnetic (EM) pump has been designed and implemented as a means of actively controlling the NaK flow rate through the system and an EM flow sensor is employed to monitor the developed flow rate. These components allow for the matching of the flow rate conditions in test loops with those that would be found in a full-scale surface-power reactor. The design and operating characteristics of the EM pump and flow sensor are presented. In the EM pump, current is applied to a set of electrodes to produce a Lorentz body force in the fluid. A measurement of the induced voltage (back-EMF) in the flow sensor provides the means of monitoring flow rate. Both components are compact, employing high magnetic field strength neodymium magnets thermally coupled to a water-cooled housing. A vacuum gap limits the heat transferred from the high temperature $\mathrm{NaK}$ tube to the magnets and a magnetically-permeable material completes the magnetic circuit. The pump is designed to produce a pressure rise of 5 psi, and the flow sensor's predicted output is roughly $20 \mathrm{mV}$ at the loop's nominal flow rate of $0.5 \mathrm{GPM}$.
\end{abstract}

Keywords: non-nuclear testing; electromagnetic pump; electromagnetic flow sensor; liquid-metal coolant.

PACS: 28.41.Fr, 47.65.-d, 07.07.Df.

\section{INTRODUCTION}

The Early Flight Fission-Test Facility (EFF-TF) group at NASA's Marshall Space Flight Center (MSFC) performs testing of representative reactor components and systems using resistively-heated elements to simulate the heat generated by a nuclear reactor. This non-nuclear testing methodology allows for the evaluation of reactor components in a realistic thermal and hydraulic environment without incorporating nuclear material into the test setup, avoiding the additional cost, time, and safety concerns that arise from such testing.

Present work being performed at MSFC is aimed at simulating affordable fission surface power (FSP) systems that could be used on the lunar surface. In this work, a eutectic mixture of sodium and potassium (NaK) is considered as the heat-transfer medium in the simulated reactor. The hot $\mathrm{NaK}$ is pumped through the system using $316 \mathrm{~L}$ stainless steel tubing. One issue with $\mathrm{NaK}$, or any alkali metal at elevated temperature, is the resistance of the various components in the system to corrosion and there is uncertainty regarding the corrosion rates of stainless steel at the operating conditions estimated for a FSP system (Zhang, 2007).

A Feasibility Test Loop (FTL) is being used to simulate possible flow conditions in a FSP system to provide a hardware-based measurement of corrosion and material transport (Godfroy, et al., 2007). Since the corrosion rate is potentially related to many of the flow parameters the apparatus is designed to operate at temperatures, temperatures differences, flow velocities, and L/D ratios that are representative of a reactor system. To achieve these conditions, the FTL is designed to operate at a flow rate of $0.5 \mathrm{GPM}$ and a maximum temperature of $880 \mathrm{~K}$. A pump pressure head of approximately 5 psi is calculated to be required to maintain the flow rate. 
Active pumping is required for the FTL to match the flow conditions found in a nuclear reactor system. To achive this flow a direct current (DC) electromagnetic (EM) pump (c.f. Polzin, 2007, Polzin, 2007a, Polzin and Markusic, 2007) was implemented. In addition, an EM flow meter (Markusic, et al., 2005) was used to directly monitor the flow rate in the FTL to provide feedback control of the pumping. In this paper, described are the construction of an EM pump and the flow meter, which were made compact through the use of permanent, rare-Earth magnets. In the next section, reviewed is the general scaling relations governing the operation of both components and provide an outline of the component materials and design choices. The magnetic field characteristics and the performance of the pump and flow meter are estimated in the following section.

\section{HARDWARE DESIGN}

A brief discussion regarding the theoretical scaling of EM pump and flow sensor performance is presented. This is followed by a description of the actual design of the hardware implemented in the present FTL.

\section{Pump and Flow Sensor Performance Scaling}

An idealized schematic of a DC EM pump is presented in Fig. 1, left panel. The liquid metal flows though a channel of width $w$, length $l$, and height $s$. The magnetic field $B$ and current $I$ are perpendicular to the flow (and each other) and are uniform over the indicated faces. When current flows between the electrodes, a Lorentz body force

$$
\vec{f}_{L}=\vec{j} \times \vec{B}
$$

is exerted on the fluid in the direction of the flow vector. Assuming a uniform current density between the electrodes, the pressure $P$ produced by the pump (neglecting losses) is given as

$$
P=I \frac{B}{s} .
$$

In Eq. (2), the pressure developed by the pump is only a function of the channel height, magnetic field strength, and total current. Material properties, specifically the conductivity of the fluid, are important only when considering the electric potential required to drive current through the pump.
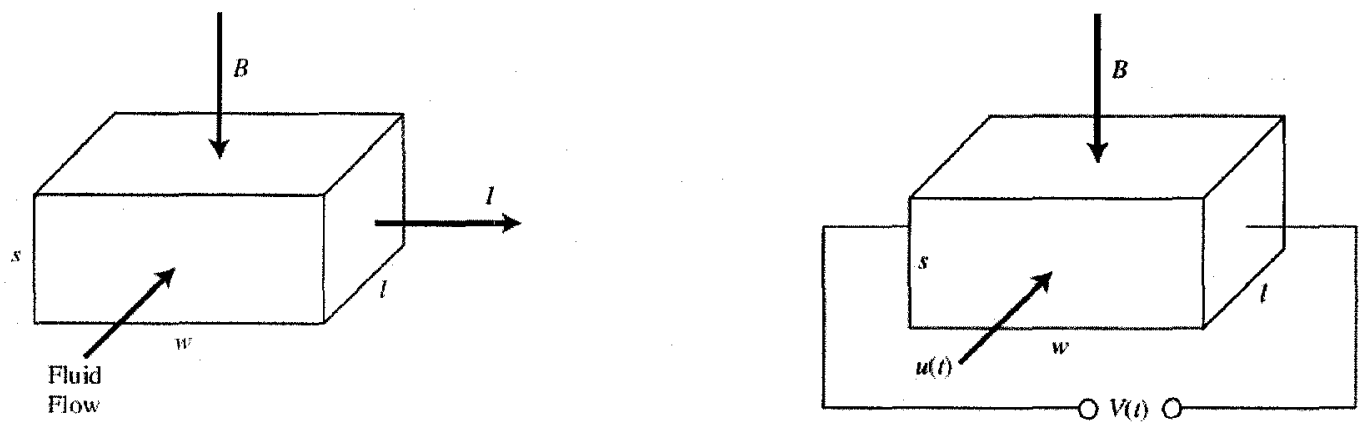

FIGURE 1. Idealized schematic of an electromagnetic pump (left) and flow sensor (right).

A DC EM flow sensor (shown schematically in the right panel of Fig. 1) is similar to an EM pump in both construction and operation. The primary difference is that instead of imposing a transverse current through the electrodes, a voltage is measured in the direction perpendicular to the flow and the applied magnetic field. When an electrically conductive medium (a conducting liquid in this case) moves transverse to an applied field, a back-EMF is induced according to the vector equation 


$$
\vec{E}=\vec{u} \times \vec{B}
$$

Integrating the electric field over the width $w$, the voltage induced across the channel is equal to

$$
V=\frac{\dot{m}}{\rho} \frac{B}{s}=\dot{V} \frac{B}{s},
$$

where $\dot{m}$ is the mass flow rate through the sensor and $\dot{V}$ is the corresponding volumetric flow rate. As in the pump, the voltage output by the flow sensor is linearly dependent on the ratio $\mathrm{B} / \mathrm{s}$. In addition, once the flow meter is calibrated, it should be relatively simple to use since the output voltage scales linearly with the volumetric flow rate.

\section{Implementation}

It is apparent from Eq. (2) that the pump will be optimized (produce the greatest pressure head for the least amount of current) when the ratio of $B / s$ is maximized. Additionally, it is seen in Eq. (4) that the voltage output for a given flow rate is increased with an increasing value of $B / s$. This ratio was maximized in both the EM pump and flow meter and, for the sake of simplicity, the same design was chosen for both components.

Renderings of the EM pump are shown in Fig. 2 (there is no real difference between the pump and flow sensor, so only the pump design will be discussed here). The hot liquid $\mathrm{NaK}$ is conducted through a 0.5 " diameter stainless steel tube that has been flattened inside the pump. The flattened section has permanent neodymium rare-Earth magnets on each side, imposing an applied field in the vertical direction. Flattening the tube not only cuts down the value of $s$, but it also allows us to bring the magnet faces as close together as possible to increase the value of $B$. The neodymium magnets have a relatively low Curie temperature $(80-100 \mathrm{C})$, so they are held a small distance away from the flattened tube, which has a temperature of up to $880 \mathrm{~K}$. The resulting vacuum gap allows only radiative heat transfer from the steel to the magnet face. The water-cooled copper blocks serve to conductively transfer heat from the magnets. In addition, it also provides the structure required to keep the magnets a given distance apart from each other. The magnetic circuit of the pump is completed using pieces of FluxTrol, which is a magnetically permeable material. Metal bus bars (electrodes) are welded to the sides of the flattened tube to introduce current to the pump. The magnets are much longer than the electrodes in the direction of fluid flow to keep the field strength relatively constant over their entire electrode width. The only difference between the pump and flow sensor is that thin metal weld rods are used as electrodes instead of flat, rectangular bus bars.
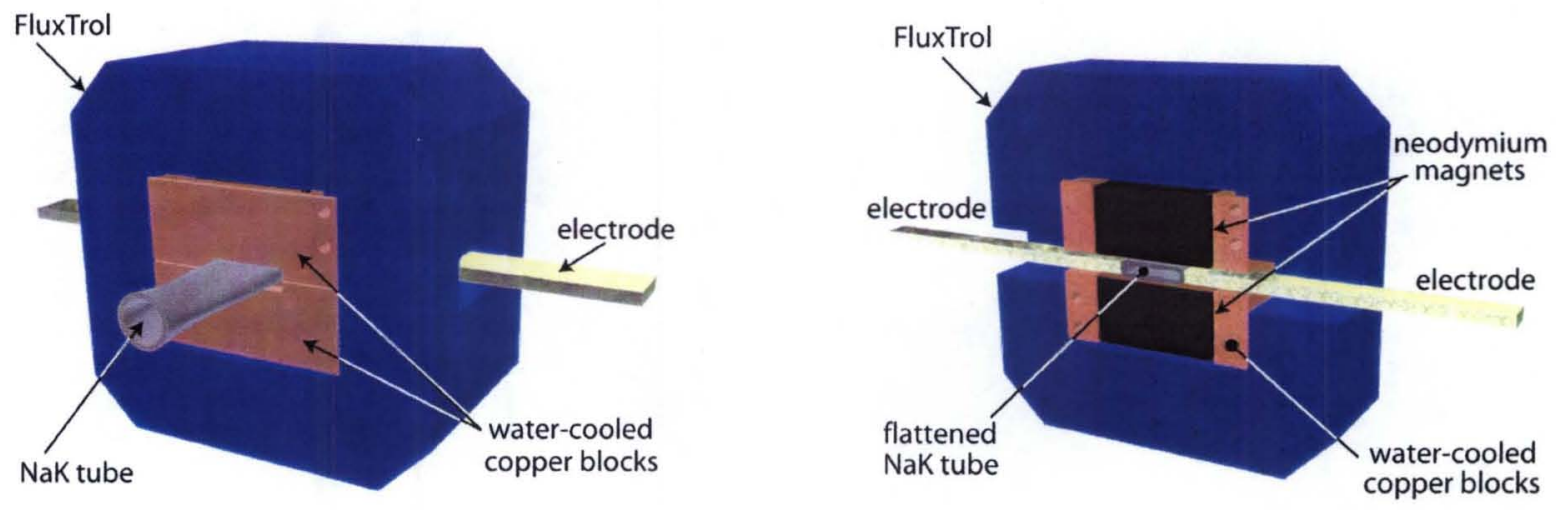

FIGURE 2. Renderings of the EM pump: external view (left) and internal cross-sectional view (right). 


\section{FIELD AND PERFORMANCE CALCULATIONS}

In this section, the field strength is computed using a magnetostatic solver, and then the value of $B$ in the channel is used to estimate the performance of the EM pump and flow meter.

\section{Field Calculations}

The magnetic field is found using the two-dimensional magnetostatic solver package (Quickfield). This solver has the capability of modeling materials, like the FluxTrol and the neodymium magnets, which possess non-linear $B-H$ characteristics. The $B-H$ curves for each material in the direction of the magnetic flux are given in Fig. 3 . While these materials are actually anisotropic, it is acceptable to use only one $B-H$ curve for each since the magnetic field in each component will only be aligned in the direction corresponding to these curves.
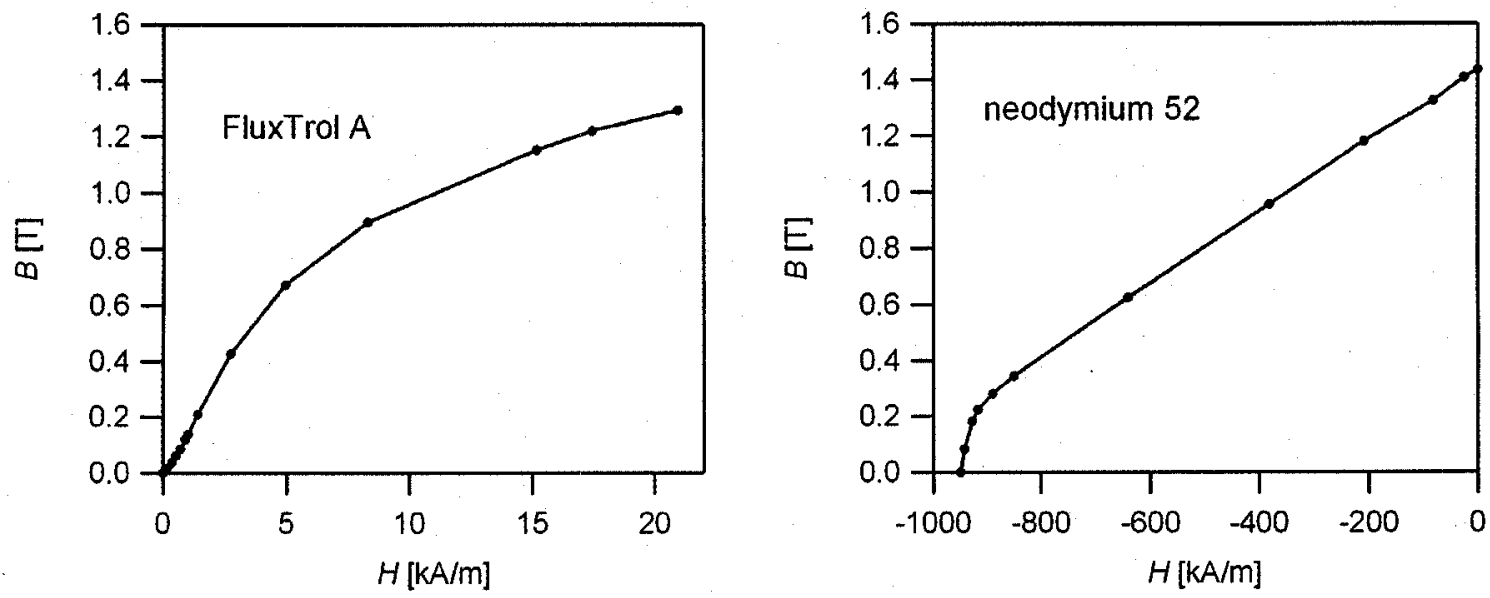

FIGURE 3. $B-H$ characteristics in the direction of the magnetic field for FluxTrol A (left) and neodymium 52 (right).

A resulting solution from the magnetostatic model is presented in Fig. 4. The average value of $B$ inside the NaK channel is calculated as $\sim 1.05 \mathrm{~T}$. In addition, the modeling shows that the magnetic field lines through the $\mathrm{NaK}$ are almost completely oriented in the vertical direction, and the field strength is approximately uniform in the gap. This yields a field topology that is approximately equivalent to that assumed in the theoretical analysis associated with Fig. 1. The FluxTrol serves to capture the field, keeping it contained within the component. This not only helps to increase the field in the $\mathrm{NaK}$ channel, but it also keeps the field from potentially interfering with other components located near the pump or flow sensor.

\section{Pump and Flow Meter Performance}

The performance of the EM pump and flow meter can be estimated using Eqs. (2) and (4), respectively. From the magnetostatic model, $B=1.05 \mathrm{~T}$. In addition, the NaK tube is flattened so that the dimension $s$ is $1 / 16^{\prime \prime}$.

The pump pressure head produced for a given input current is presented in the left-hand panel of Fig. 5. The variable $I_{\text {pump }}$ represents the total current sent to the pump from the power supply. The solid line is the pressure produced by the pump assuming all the current from the power supply actually conducts through the $\mathrm{NaK}\left(I_{\mathrm{NaK}}=\right.$ $I_{\text {pump }}$ ). In reality, however, some current sent to the pump will conduct only through the stainless steel walls of the tube containing the NaK. The ratio of this parallel shunt current, $I_{\text {shunt }}$, to $I_{\mathrm{NaK}}$ scales with the resistance ratio $R_{\mathrm{Nak}} / R_{\text {shunt }}$, where these are the lumped element resistances presented to the power supply by each material. The dotted line in the left-hand panel of Fig. 5 represents a case where the value of $R_{\text {Nak }} / R_{\text {shunt }}$ is estimated equal to unity. This yields $I_{\mathrm{NaK}}=I_{\text {shunt }}=I_{\text {pump }} / 2$. The figure demonstrates that the performance of the pump decreases linearly with 
increasing shunt current. In this case, which is representative of actual pump operation, the power supply must provide $110 \mathrm{~A}$ to produce the required $5 \mathrm{psi}$ pressure head.

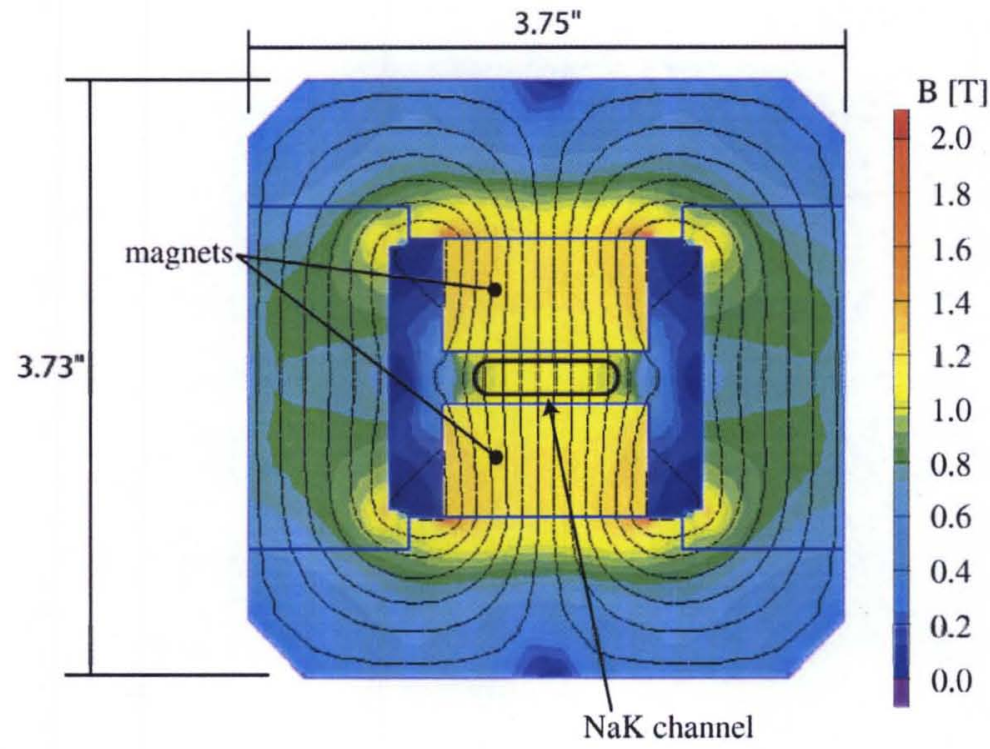

FIGURE 4. Magnetostatic simulation result for the geometry shown in Fig. 2. The average $\boldsymbol{B}$ in the channel is $\sim 1.05 \mathrm{~T}$.
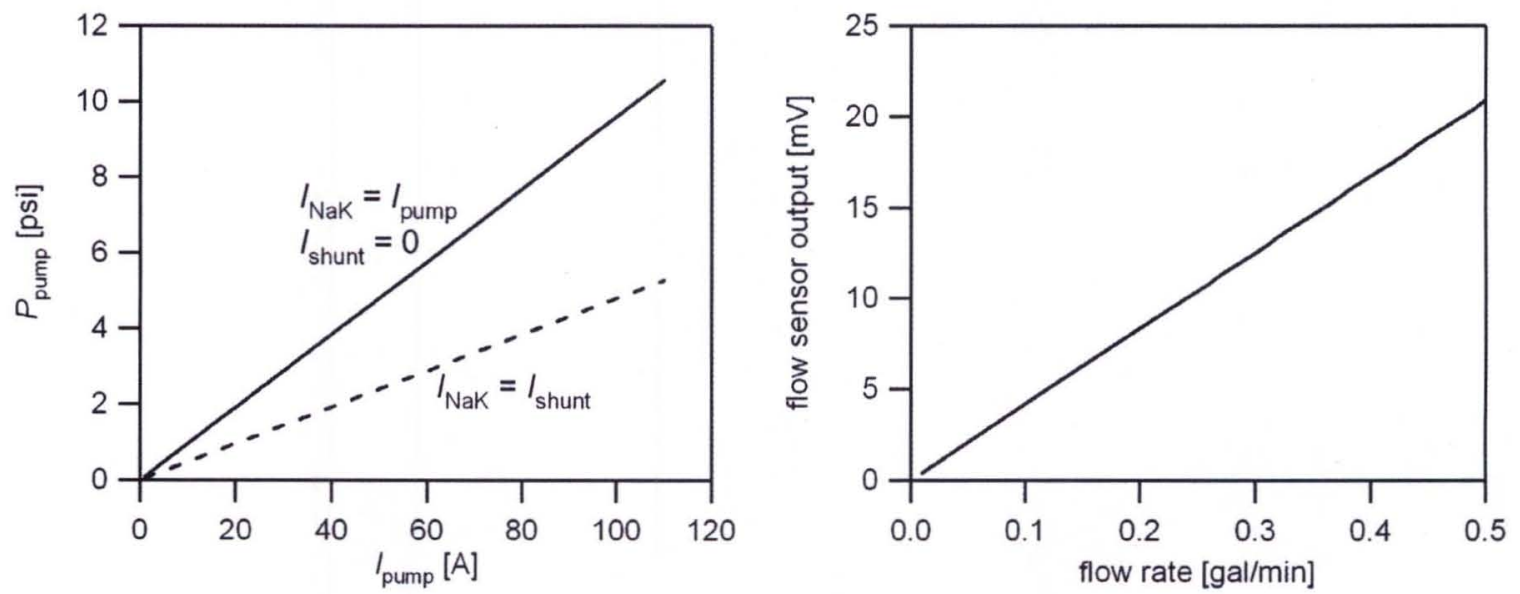

FIGURE 5. (left) Theoretical EM pump pressure head produced as a function of input current (Eq. (2)) when all current enters the $\mathrm{NaK}\left(I_{\mathrm{NaK}}=I_{\text {pump }}, I_{\text {shunt }}=0\right)$ and when half the current going into the pump is shunted around the NaK $\left(I_{\mathrm{NaK}}=I_{\text {shunt }}\right)$, performing no work. (right) Theoretical voltage output of the EM flow sensor as a function of volumetric flow rate (Eq. (4)). $B=1.05 \mathrm{~T}$ and $s=1 / 16$ " for both the pump and flow meter.

The output of the flow meter as a function of volumetric flow rate (throughput) is presented in the right-hand panel of Fig. 5. The voltage output is linear with flow rate, which is expected based on Eq. (4). In addition, for the maximum flow rate expected in the FTL $(0.5 \mathrm{GPM})$ the flow sensor output voltage is just above $20 \mathrm{mV}$. While some assumptions were made during the theoretical analysis regarding the field configuration, it is still expected that the output voltage level will be on the order of that predicted using Eq. (4). 


\section{CONCLUSIONS}

In this paper were presented a DC EM pump and flow sensor designed for inclusion in a test loop that aims at evaluating the long-term effects of flowing, hot $\mathrm{NaK}$ on various components and subsystems that might comprise a lunar nuclear reactor system. The pump is incorporated to provide active control of the flow rate, which must be kept at a given level to properly match the operational conditions that may affect the corrosion rate in the test loop with that found in a real reactor system. The EM flow sensor provides a means for monitoring the flow rate, and can be used to provide feedback control to the pump. The pump and flow sensor designed for the FTL are fundamentally the same, with emphasis placed on creating a design that maximized the magnetic field within the $\mathrm{NaK}$. The field is increased by flattening the tube containing the $\mathrm{NaK}$ to minimize the gap between the neodymium magnet faces and by using magnetic pole pieces (FluxTrol) to contain the magnetic flux within the components. The use of rare-Earth, neodymium magnets is made possible by a vacuum gap that allows only radiative heat transfer from the hot, NaK-filled tube to the magnet faces, and the magnets are thermally-coupled to water-cooled copper blocks. A magnetostatic simulation showed that through the NaK-filled tube was a nearly-uniform field of $1.05 \mathrm{~T}$. In the case where the current to the pump is equally partitioned between the $\mathrm{NaK}$ and the stainless steel containment tube, the pump produces the required pressure head of 5 psi for an input (power supply) current of 110 A. The predicted output of the flow sensor at the nominal flow rate of $0.5 \mathrm{GPM}$ is roughly $20 \mathrm{mV}$.

\section{NOMENCLATURE}

$\begin{array}{llll}\vec{B}, B & \text { magnetic induction }[\mathrm{T}] & P & \text { pressure }[\mathrm{Pa}] \\ \vec{E} & \text { electric field }[\mathrm{V} / \mathrm{m}] & R & \text { electrical resistance }[\Omega] \\ \overrightarrow{f_{L}} & \text { Lorentz body force }\left[\mathrm{N} / \mathrm{m}^{3}\right] & \rho & \text { mass density }\left[\mathrm{kg} / \mathrm{m}^{3}\right] \\ H & \text { magnetic intensity }[\mathrm{A} / \mathrm{m}] & s & \text { height dimension }[\mathrm{m}] \\ I & \text { current }[\mathrm{A}] & \vec{u} & \text { velocity }[\mathrm{m} / \mathrm{s}] \\ \vec{j} & \text { current density }\left[\mathrm{A} / \mathrm{m}^{2}\right] & V & \text { voltage }[\mathrm{V}] \\ l & \text { length dimension }[\mathrm{m}] & \dot{V} & \text { volumetric flow rate }\left[\mathrm{m}^{3} / \mathrm{s}\right] \\ \dot{m} & \text { mass flow rate }[\mathrm{kg} / \mathrm{s}] & w & \text { width dimension }[\mathrm{m}]\end{array}$

\section{ACKNOWLEDGMENTS}

NASA's Exploration Technology Development Program supported the work described within this paper, in whole or part, as part of the program's technology development and evaluation activities. Any opinions expressed are those of the author(s) and do not necessarily reflect the views of the Exploration Technology Development Program or NASA.

\section{REFERENCES}

Godfroy, T.J., Pearson, J.B., Polzin, K.A., Reid, R.S., and Webster, K.L., "FTL-1 Feasibility Test Loop Design and Construction," in proceedings of the American Nuclear Society Space Nuclear Conference 2007, Boston, MA, 2007. Paper 2029.

Markusic, T.E., Polzin, K.A., Stanojev, B.J., Dodson, C., Dehoyos, A., "Liquid Metal Flow Sensors for Electric Propulsion," in JANNAF JPM-LPM-SPS Joint Propulsion Meeting, Monterey, CA, Dec. 2005.

Polzin, K.A., "Liquid Metal Pump Technologies for Nuclear Surface Power," in proceedings of the American Nuclear Society Space Nuclear Conference 2007, Boston, MA, 2007. Paper 2002.

Polzin, K.A., Liquid-Metal Pump Technologies for Nuclear Surface Power, NASA TM-2007-214851, NASA-Marshall Space Flight Center, Huntsville, AL, 2007a.

Polzin, K.A. and Markusic, T.E., "Electromagnetic Pumps for Liquid Metal-Fed Electric Thrusters," Journal of Propulsion and Power, accepted for publication, Aug. 2007. 
Zhang, J and Marcille, T.F., "Assessment of Corrosion in Liquid NaK System," in proceedings of the International Congress on Advances in Nuclear Power Plants, Nice, France, 2007. Paper 7407. 